AIP Applied Physics

\title{
Understanding the operation of quantum dot intermediate band solar cells
}

\author{
A. Luque, P. G. Linares, E. Antolín, I. Ramiro, C. D. Farmer et al.
}

Citation: J. Appl. Phys. 111, 044502 (2012); doi: 10.1063/1.3684968

View online: http://dx.doi.org/10.1063/1.3684968

View Table of Contents: http://jap.aip.org/resource/1/JAPIAU/v111/i4

Published by the American Institute of Physics.

\section{Related Articles}

Radial p-n junction macroporous silicon solar cell on p-type upgraded metallurgical-grade silicon substrate J. Renewable Sustainable Energy 4, 063108 (2012)

Surface-plasmon enhanced absorption in organic solar cells by employing a periodically corrugated metallic electrode

Appl. Phys. Lett. 101, 163303 (2012)

Surface-plasmon enhanced absorption in organic solar cells by employing a periodically corrugated metallic electrode

APL: Org. Electron. Photonics 5, 234 (2012)

Development of pulsed laser deposition for CdS/CdTe thin film solar cells

Appl. Phys. Lett. 101, 153903 (2012)

Technology-compatible hot carrier solar cell with energy selective hot carrier absorber and carrier-selective contacts

Appl. Phys. Lett. 101, 153901 (2012)

\section{Additional information on J. Appl. Phys.}

Journal Homepage: http://jap.aip.org/

Journal Information: http://jap.aip.org/about/about_the_journal

Top downloads: http://jap.aip.org/features/most_downloaded

Information for Authors: http://jap.aip.org/authors

\section{ADVERTISEMENT}

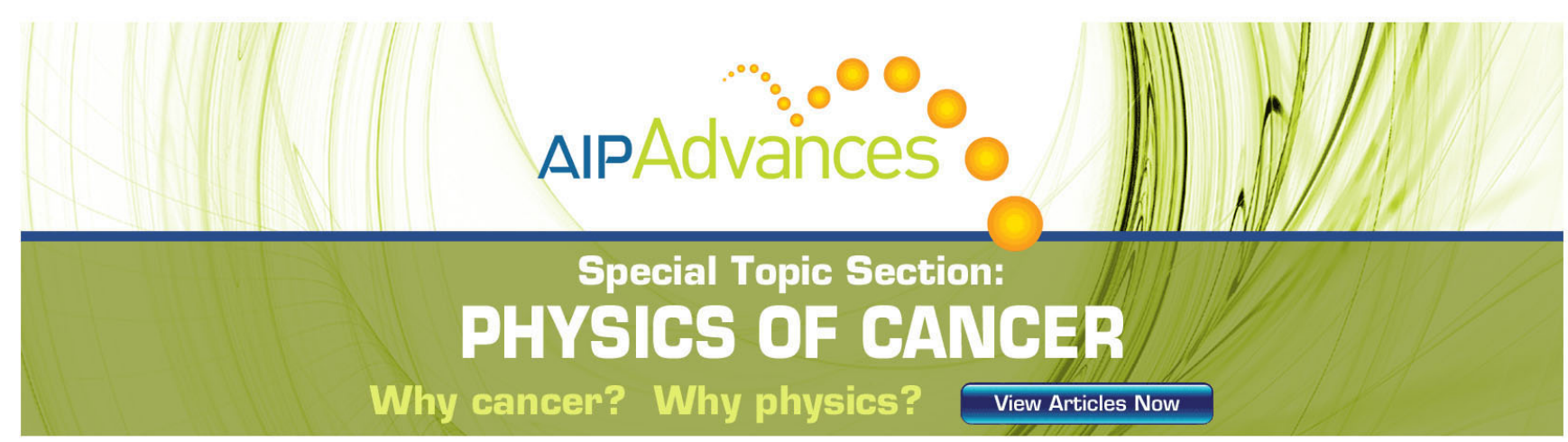




\title{
Understanding the operation of quantum dot intermediate band solar cells
}

\author{
A. Luque, ${ }^{1}$ P. G. Linares, ${ }^{1, a)}$ E. Antolín, ${ }^{1}$ I. Ramiro, ${ }^{1}$ C. D. Farmer,${ }^{2}$ E. Hernández, ${ }^{1}$ \\ I. Tobías, ${ }^{1}$ C. R. Stanley, ${ }^{3}$ and A. Martí ${ }^{1}$ \\ ${ }^{1}$ Instituto de Energía Solar, Universidad Politécnica de Madrid, E.T.S.I. de Telecomunicación, \\ Ciudad Universitaria s/ $n 28040$ Madrid, Spain \\ ${ }^{2}$ Kelvin Nanotechnology Ltd, Rankine Building, Oakfield Avenue, Glasgow G12 8LT, United Kingdom \\ ${ }^{3}$ School of Engineering, University of Glasgow, Oakfield Avenue, Glasgow, G12 8LT, United Kingdom
}

(Received 11 November 2011; accepted 12 January 2012; published online 17 February 2012)

\begin{abstract}
In this paper, a model for intermediate band solar cells is built based on the generally understood physical concepts ruling semiconductor device operation, with special emphasis on the behavior at low temperature. The model is compared to $J_{\mathrm{L}}-V_{\mathrm{OC}}$ measurements at concentrations up to about 1000 suns and at temperatures down to $20 \mathrm{~K}$, as well as measurements of the radiative recombination obtained from electroluminescence. The agreement is reasonable. It is found that the main reason for the reduction of open circuit voltage is an operational reduction of the bandgap, but this effect disappears at high concentrations or at low temperatures. (C) 2012 American Institute of Physics. [doi:10.1063/1.3684968]
\end{abstract}

\section{INTRODUCTION}

The intermediate band (IB) solar cell ${ }^{1,2}$ has an allowed electron energy band within the bandgap in order to obtain current from sub-bandgap photons. Provided the electrons in the IB are characterized by a quasi-Fermi level (QFL) that is independent of those in the conduction band (CB) and in the valence band (VB), the voltage of the cell can be determined by the CB and VB QFL positions. In this way the solar cell can deliver electrons at a voltage that is above the energy of the less energetic photon that generates current. The thermodynamics requires ${ }^{3,4}$ more than one low-energy photon to cooperate in pumping the electron in order to deliver this high voltage. The confined levels of quantum dots (QDs) may be used as an IB. ${ }^{5}$ The attractiveness of the IB solar cell lies in the fact that its detailed balance efficiency limit, calculated following the lines of the elegant Shockley and Queisser $^{6}$ (SQ) paper, is $63 \%,{ }^{1}$ compared with $41 \%$ in the SQ model.

QD IB solar cells have been manufactured (by several researchers $)^{7-10}$ and characterized ${ }^{11-13}$ at low temperature and high irradiance. The additional sub-bandgap current has been observed by many researchers, and the preservation of voltage has been proven at low temperature. ${ }^{11}$ Several papers have modeled the operation of an intermediate band solar cell including drift-diffusion mechanisms. ${ }^{14-17}$ The purpose of this paper is to model the measured low temperature behavior by using the standard knowledge of device physics. This will help us to better understand this device and ways of improving it.

In ideal solar cells, the superposition principle is fulfilled. This fact is widely used to draw plots of the dark current that are superimposed on the photogenerated currentopen circuit voltage $\left(J_{\mathrm{L}}-V_{\mathrm{OC}}\right)$ plots at several irradiances. With this approach the effects of the series resistance disap-

\footnotetext{
a) Author to whom correspondence should be addressed. Electronic mail: p.garcia-linares@ies-def.upm.es.
}

pear, and a signature of the recombination in the cell is cleanly obtained. A simplified equivalent circuit of an IB solar cell is presented in Fig. 1. ${ }^{7}$ In theory, the superposition principle does not hold in this structure; it is a simple circuital exercise to verify that the current through diodes in the dark for a certain applied external voltage is not the same as that when illumination is applied with the cell in an open circuit. However, if the current sources $\mathrm{J}_{\mathrm{L} 2}$ and $\mathrm{J}_{\mathrm{L} 3}$ are negligible, as is unfortunately the case in our IB solar cells, the superposition holds again.

We study in this paper the $J_{L^{-}} V_{O C}$ plots experimentally obtained ${ }^{18}$ in sample solar cells in irradiance ranges up to about 1000 suns and temperatures down to $20 \mathrm{~K}$, and we try to interpret them based on standard knowledge of device physics. With this procedure we can almost totally ignore the complex transport phenomena taking place at low temperature, not only in the solar cell itself, but also in contacts that might have a non-Ohmic behavior under these conditions. Furthermore, measurements ${ }^{19}$ of the radiative and non-radiative recombination at several temperatures also are integrated into our model.

Two types of GaAs-based solar cells grown in a molecular beam epitaxy reactor have been used for the experimental part of this work: a QD-intermediate band solar cell (IBSC) device containing 30 stacked InAs/GaAs QD layers that are meant to act as the IB material, and a conventional $p$ - $n$ structure as a reference cell. The QD-IBSC structure is the same as that labeled SB in Ref. 12, and details about its fabrication are given in that reference. Figure 2 represents the layer structures of both cells, together with the composition, doping, and thickness data corresponding to each layer. The left-hand side of the figure, which corresponds to the GaAs reference cell, first shows a $625 \mu \mathrm{m}$ thick $n$-type layer corresponding to the GaAs wafer on top of which the device was grown. The first epitaxially grown layer corresponds to the buffer layer, which is meant to smooth the wafer surface. Then, an $n^{+}-\mathrm{Al}_{0.2} \mathrm{Ga}_{0.8} \mathrm{As}$ layer is used as a back-surfacefield (BSF), and after it an $n$-type $2 \times 10^{17} \mathrm{~cm}^{-3} 3.1 \mu \mathrm{m}$ thick layer is grown as the base of the solar cell. The $p-n$ junction 


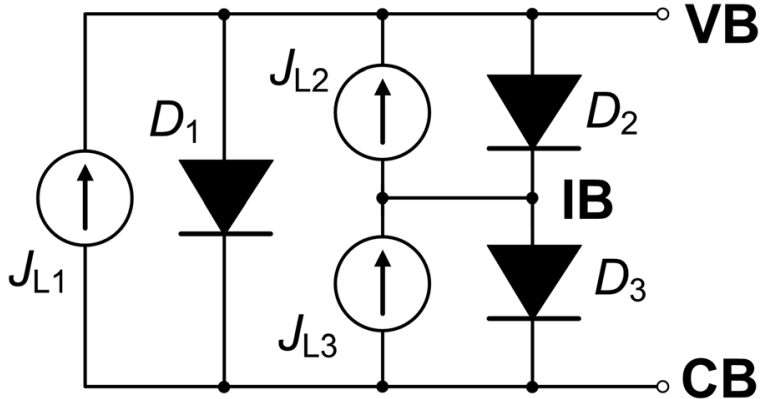

FIG. 1. Simplified equivalent circuit of the IB solar cell. Note that the electrons flow opposite to the current.

is then created, with the following $0.9 \mu \mathrm{m}$ thick $p$-type GaAs layer corresponding to the emitter. Finally, a $p^{+}$-AlGaAs window layer, a highly doped GaAs contact layer, and an antireflective coating complete the reference cell structure. The right-hand side of Fig. 2 shows a sketch of the IBSC layer structure, which is basically similar to that of the reference cell, except for the inclusion of the QD-IB region. This region contains a stacking of 30 self-assembled InAs QD layers built up via the Stranski-Krastanov growth mode and separated by thick GaAs spacers, which are meant to dilute the accumulated vertical strain and thus prevent dislocations. This QD stacking strategy is an alternative to the strain compensation technique ${ }^{7-10}$ and also prevents carrier tunneling between contiguous QD layers. ${ }^{12}$ A quaternary $\mathrm{In}_{0.2} \mathrm{Al}_{0.2} \mathrm{Ga}_{0.6} \mathrm{As}$ capping was added on top of the InAs QDs to produce a band diagram more adequate for IBSC applications. ${ }^{12} \mathrm{Si} \delta$-doping was added within each of the QD layers to partially fill the IB with electrons. ${ }^{20}$ Based on the data in the figure, the average doping density is $3.75 \times 10^{15} \mathrm{~cm}^{-3}$, calculated as the number of dopants per unit of area divided by the spacer thickness.
A qualitative band diagram is presented in Fig. 3, showing the QD potential wells and the approximate band diagram with neutral and space charge zones for the QD region.

The structure of this paper is as follows: First, we derive expressions for all the recombination processes contributing to the dark current (with emphasis on their temperature dependence). This includes non-radiative recombination components of the ordinary cell (Sec. II), non-radiative recombination components for the QD-IB solar cell (Sec. III), and radiative currents (Sec. IV). In Sec. V, we combine those expressions to build a model for the total current in the QD-IB solar cell in the dark as a function of the applied voltage. The results of this model are compared to experimental data in Sec. VI, and conclusions are drawn in Sec. VII.

\section{CLASSICAL EXPRESSIONS OF THE EMITTER OR BASE CURRENT}

IB solar cells and the ordinary test cells used for comparison have similar p-emitters. Only the test cell has an n-base zone bounded in the back by a hetero-junction meant to suppress the back recombination. It is assumed that no noticeable recombination is produced in the heterostructure forming the BSF layer. This n-base is substituted in the IB cells by an almost intrinsic region in which the QDs are analyzed. This intrinsic region is studied in Sec. III A.

\section{A. Neutral zones}

The recombination behavior of ordinary emitters and bases in solar cells is well known. It involves neutral zone recombination and space-charge recombination. Here we collect the expressions of those recombination currents using the nomenclature for an $n$-region. The expressions are determined by the parameters of the minority carriers-holes in
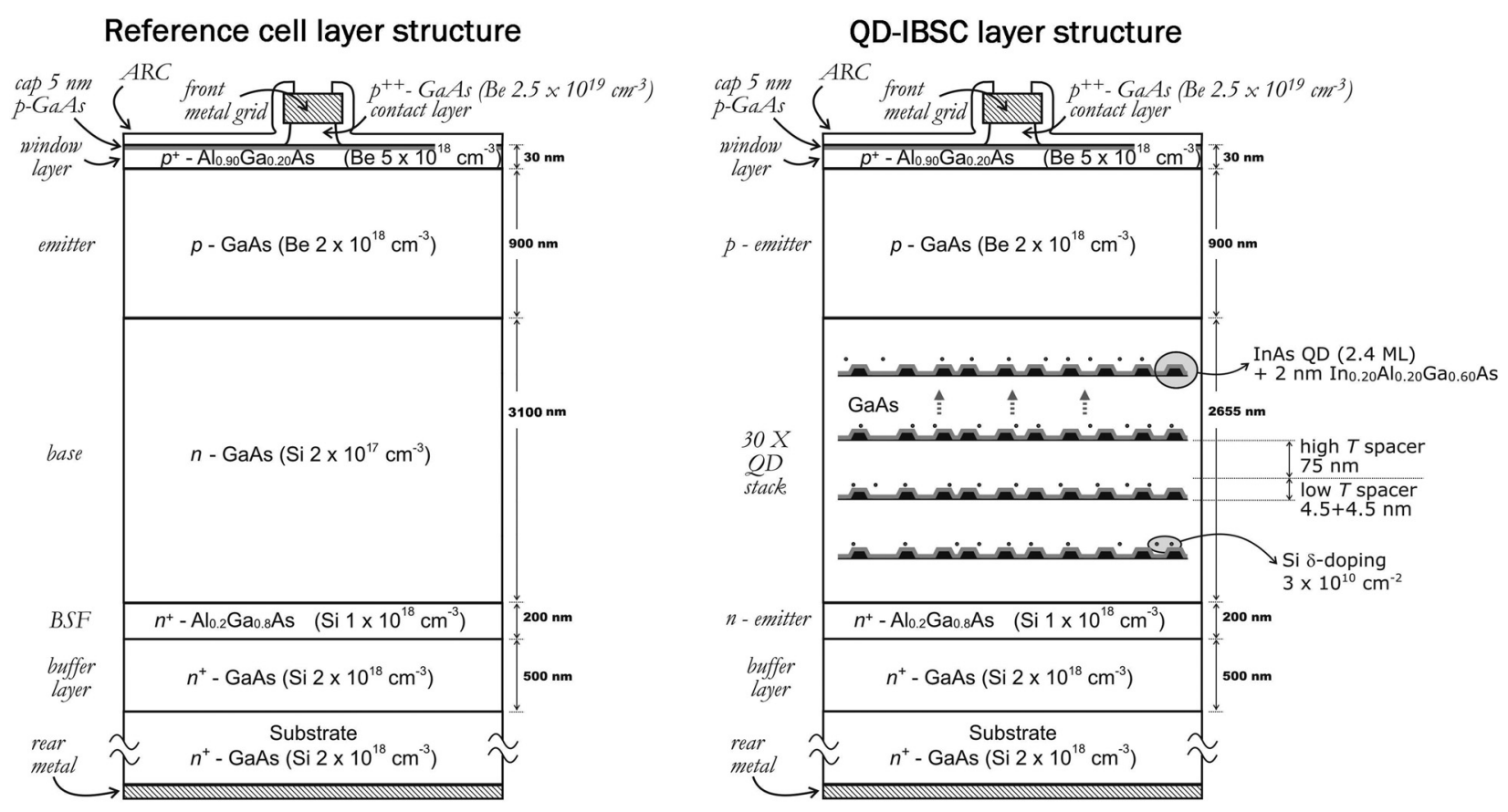

FIG. 2. The left-hand side of the figure shows a sketch of the layer structure corresponding to the GaAs reference cell. The right-hand part of the figure shows the layer structure of the InAs/GaAs QD-IBSC including the 30 stacked QD layers. 

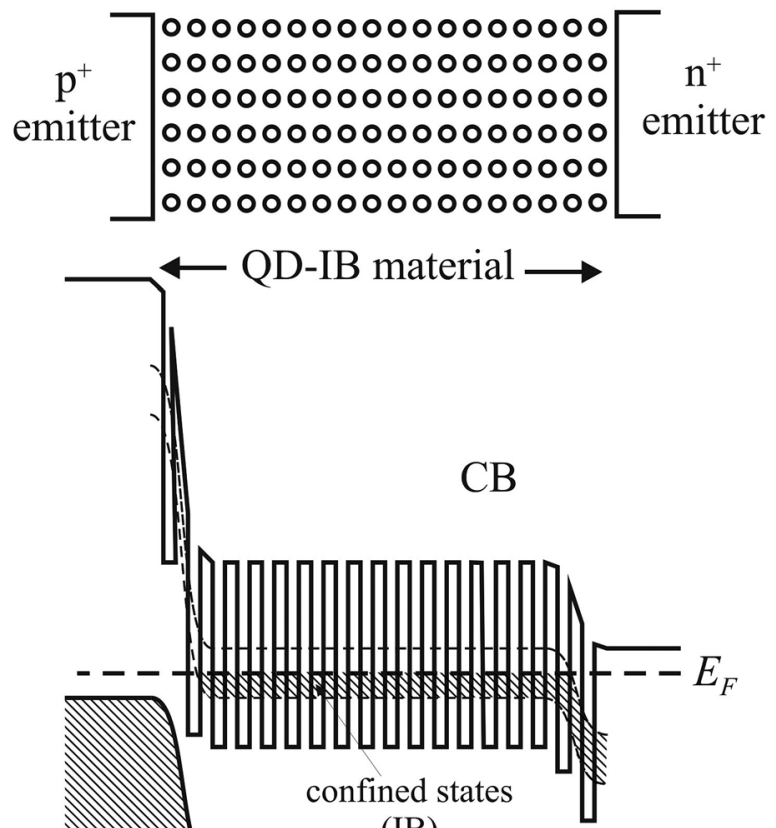

(IB)

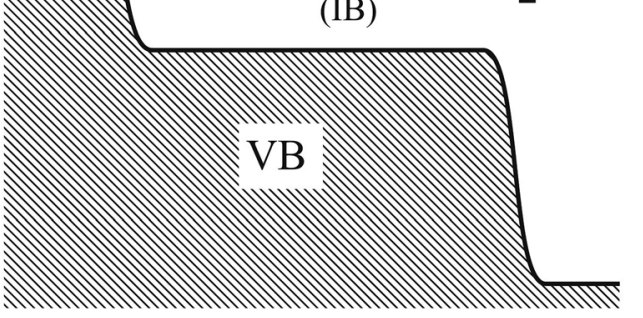

FIG. 3. Simplified band diagram of a quantum dot intermediate band solar cell showing the conduction, valence, and intermediate bands.

this case-which we denote by the sub-index $p$. To study the $p$-emitter, the expressions are kept the same, but the subindex $p$ has to be changed to $n$, and other parameters have to be interchanged as is standard in semiconductor physics (e.g., $N_{\mathrm{A}}$ for $N_{\mathrm{D}}$ ).

The neutral zone recombination current in low injection is the product of the diode factor $[\exp (e V / k T)-1]$ times the saturation current, given by $e p_{e q} L_{p} / \tau_{p}$ for long diodes and $e p_{e q} W / \tau$ for short diodes, with zero surface recombination in the back side (the one farthest from the junction), which, thanks to the hetero-structured BSF, is the only one of interest here ( $W$ is the region width). These are asymptotic expressions; the exact expression is given, e.g., in Ref. 21 (p. 20). In particular, $p_{e q}$ is the minority carrier equilibrium concentration in an $\mathrm{n}$ region, $L_{p}=\sqrt{D_{p} \tau_{p}}$ is the diffusion length, $D_{p} \cong \mu_{p} k T / e$ (the equality holds for non-degenerated carriers, which is usually the case for minority carriers) is the diffusion coefficient, $\mu_{p}$ is the mobility, $\tau_{p}=1 / v_{t h} \sigma_{p} N_{t}$ is the Shockley-Read-Hall (SRH) lifetime, $v_{t h}=\sqrt{3 k T / m_{p}}$ is the thermal velocity, $\sigma_{p}$ is the recombination capture section, $N_{t}$ is the density of recombination centers, and $m_{p}$ is the effective mass.

We are especially interested in the temperature evolution of these terms; $p_{e q}$ is strongly variable according to $p_{e q}=N_{v} \exp \left(\left(E_{v}-E_{F}\right) / k T\right)$, where $N_{v}$ is the equivalent density of states in the valence band, proportional (like the equivalent density in the conduction band $N_{c}$ ) to $T^{3 / 2}, E_{v}$ is the top of the valence band, and $E_{F}$ is the equilibrium Fermi level. However, in our calculations we have considered the majority carriers as partially degenerate, and therefore the Fermi level position has been calculated by solving the equation (neglecting the degeneracy of the doping level)

$$
\frac{N_{D} / N_{c}(T)}{\exp \left(u_{F}+E_{D} / k T\right)+1}=\frac{2}{\sqrt{\pi}} \int_{0}^{\infty} \frac{\sqrt{\varepsilon} d \varepsilon}{\exp \left(\varepsilon-u_{F}\right)+1},
$$

where $E_{D}$ is the absolute value of the distance between the donor level and the conduction band and $u_{F}=\left(E_{F}-E_{c}\right) / k T$. It is remembered that $N_{c}$ is proportional to $T^{3 / 2}$ in the preceding formula.

For non-degenerate minority carriers (when the voltage is below the bandgap by about $3 k T / e$ ), we can write the recombination current as

$$
\begin{aligned}
J_{\text {em,base }} & \cong K_{01} \exp \left(\left(e V-E_{g}\right) / k T\right), \\
K_{01} & =\frac{e N_{v} \exp \left(-u_{F}\right) / \tau_{p}}{1 / W+1 / L_{p}}=\frac{e N_{v} \exp \left(-u_{F}\right) W_{e q}}{\tau_{p}} .
\end{aligned}
$$

The expression $W_{e q}=\left(1 / W+1 / L_{p}\right)^{-1}$ is a simplification used to match the asymptotic behaviors for short and long diodes explained above. For the case of short diodes with non-degenerate majority carriers, Eq. (1) becomes $N_{D}^{+} / N_{c}=\exp \left(u_{F}\right)$. Then, for full donor ionization, the prefactor that we have termed $K_{01}$ in Eq. (2) becomes $K_{\text {approx }}=e\left(N_{v} N_{c} / N_{D}\right)\left(W / \tau_{p}\right)$, which is a frequently used expression. This expression already suggests that, all the rest of the parameters being the same, the recombination current decreases with doping of the semiconductor, although there is a limit set by the shorter lifetime of the heavily doped semiconductors.

We present in Fig. 4 the calculated value of the minority carrier diffusion length versus the absolute temperature. We can observe that the diffusion length remains almost constant with the temperature, except for very low values, at which it experiences a notable increase. When $L_{p}$ increases at very

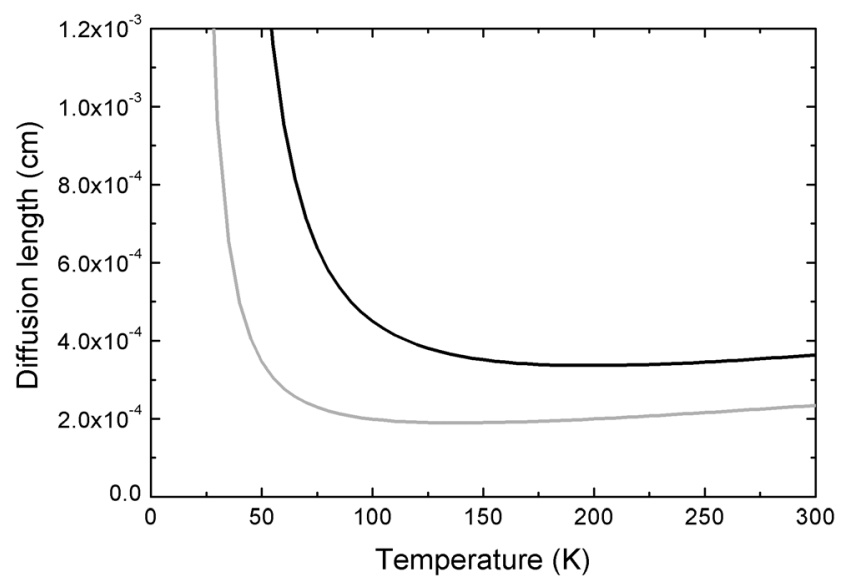

FIG. 4. Diffusion length vs temperature for the values in the table. The solid black line represents an n-type base, and the solid light gray line represents a p-type emitter. Parameters are as in Table I, except for $300 \mathrm{~K}$ lifetimes set at $0.5 \times 10^{-9} \mathrm{~s}$ and their activation energies at $0.0147 \mathrm{eV}$ for both cases. In the measured samples, the base thickness is $3.1 \times 10^{-4} \mathrm{~cm}$, and the emitter thickness is $0.9 \times 10^{-4} \mathrm{~cm}$. 
low temperatures, the short-diode approximations apply, the recombination is not dependent on $L_{p}$ anymore, and a very accurate calculation of this parameter in that range is unnecessary.

The minority carrier mobility appears in the diffusion length. At low temperature it is dominated by scattering by ionized impurities (the dopants). This scattering increases with the temperature proportionally to $T^{3 / 2}$ and decreases inversely proportionally to the ionized impurity concentration (see p. 795 in Ref. 22). Above room temperature, the mobility tends to be dominated by phonon scattering and decreases with the temperature. For very high concentrations, such as $10^{19} \mathrm{~cm}^{-3}$, we assume that at room temperature the mobility is already limited by ionized impurities and we apply the law $T^{3 / 2} / N_{D}^{+}$in our calculations. This is indeed a rough estimate, but, as said above, its influence is rather small.

The dependence of the lifetime on $T$ is given by the expression $\tau_{p}=1 / v_{t h} \sigma_{p} N_{t}$. The dependence of $v_{t h}$ has already been presented, and $N_{t}$ is independent of the temperature. We assume an Arrhenius law of the type $\sigma_{p} \propto \exp \left(-E_{\tau p} / k T\right)$ associated with the energy necessary for an electron to move from the band to the impurity (or vice versa for the $\mathrm{VB}$ ) $^{23}$ when the lattice is distorted as explained in Ref. 24. This dependence is applicable to the SRH recombination but not to the Auger recombination, which could be dominant for heavily doped materials like the emitter. However, we believe that the recombination in this region is not dominant, in particular in the case of an IBSC, so, for the sake of brevity, we omit a more detailed analysis.

By setting all the temperature dependences in Eq. (2) as explained in this section, we can calculate the $K$ pre-factors corresponding to the $p$-emitter of both cells and to the base of the test cell. The results are plotted in Fig. 5 .

The particular case in which the capture cross-section Arrhenius factor $\left(E_{\tau n}\right)$ is set to zero has also been calculated. This situation leads to a substantial increase of the recombination current at low temperature that is actually due to the

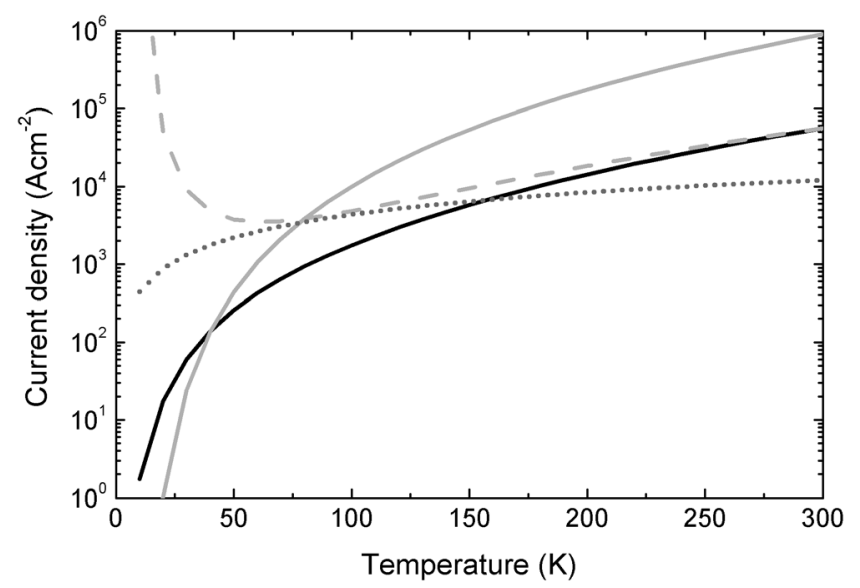

FIG. 5. Pre-factor $K$ vs $T$. Solid black line, n-type base; solid gray line, p-type emitter; dashed light gray line, p-type emitter with $E_{\tau \mathrm{n}}=0$; dotted dark gray line, radiative recombination current. Parameters are as in Table I, except for $300 \mathrm{~K}$ lifetimes set at $0.5 \times 10^{-9} \mathrm{~s}$ and their activation energies at $0.0147 \mathrm{eV}$ for all cases (excepting the dashed light gray curve activation energy, which is zero). reduction of the acceptor ionization. This means that if we assume that the capture cross-section of traps remains the same for all temperatures, then the SRH lifetime has only a weak dependence on the temperature through the thermal velocity. In that case, the thermal dependence of the recombination is dominated by the fact that at low temperature, the acceptor ionization is lost, and the material approaches an intrinsic behavior. This effect is much less perceptible in the $\mathrm{n}$-base because the energy level introduced by $\mathrm{Si}$ atoms as dopants is very close to the $\mathrm{CB}$ and remains ionized even at very low temperatures.

\section{B. Space-charge zone}

In the space-charge zone, the electron concentration varies from its value at the $n$-region to its value in the $p$-region, where the electrons are very few, and vice versa for the holes. Somewhere within the depletion region, the condition $n \tau_{n}=p \tau_{p}$ is fulfilled when the SRH recombination reaches a maximum. Its integration through the space-charge zone leads to the approximate current ${ }^{25}$

$$
\begin{aligned}
& J_{s c z} \cong K_{02} \exp \left(\left(e V-E_{g}\right) / 2 k T\right), \\
& K_{02} \cong \frac{e \sqrt{N_{c} N_{v}}}{2 \sqrt{\tau_{p} \tau_{n}}} \frac{k T}{e \mathrm{E}}
\end{aligned}
$$

where $\mathrm{E}$ is the electric field at the point at which the maximum recombination is produced. A numerical solver such as the PC1D may be used to determine this field, but a mistake in the determination of its value would be reflected only in the value for the averaged lifetime term. In other words, $\mathrm{E} \sqrt{\tau_{p} \tau_{n}}$ can be fitted as a single parameter.

The temperature dependence of all the elements in this equation has already been discussed.

\section{Bandgaps}

For $T$ in $\mathrm{K}$ and the energy in $\mathrm{eV}$, the GaAs bandgap is given by (Ref. 26, p. 15)

$$
E_{g}=1.519-\frac{5.405 \times 10^{-4} T^{2}}{T-204} .
$$

The donor and acceptor levels are given in Table I according to their distance to the nearest band and the position of the IB. These distances are considered T-independent.

Bandgap shrinkage can modify $E_{g}$ as discussed in the text. Such modifications are also considered $T$-independent. They do not affect the position of the levels.

\section{NON-RADIATIVE CURRENTS IN THE IB SOLAR CELL}

The recombination current within the QD region of the IB solar cell comprises the CB-VB recombination that is a part of the current through diode D1 in Fig. 1 (the other part corresponds to the $p$-emitter) and the current that goes through diodes D2 and D3, which represent the recombination current due genuinely to the IB itself. We examine these currents separately. 
TABLE I. Parameters in the calculations.

\begin{tabular}{|c|c|c|c|}
\hline Magnitude (at $300 \mathrm{~K}$ ) & Value & Units & $\begin{array}{c}\text { Fitting }(\mathrm{F}) \text { Measurement }(\mathrm{M}) \\
\text { Literature }(\mathrm{L})\end{array}$ \\
\hline$N_{c}$ & $4.7 \times 10^{17}$ & $\mathrm{~cm}^{-3}$ & $\mathrm{~L}$ \\
\hline$N_{v}$ & $7 \times 10^{18}$ & $\mathrm{~cm}^{-3}$ & $\mathrm{~L}$ \\
\hline$\mu_{n}$ at $10^{19} \mathrm{~cm}^{-3}$ & 1300 & $\mathrm{~cm}^{2} \mathrm{~V}^{-1} \mathrm{~s}^{-1}$ & $\mathrm{~L}$ \\
\hline$\mu_{p}$ at $10^{19} \mathrm{~cm}^{-3}$ & 60 & $\mathrm{~cm}^{2} \mathrm{~V}^{-1} \mathrm{~s}^{-1}$ & $\mathrm{~L}$ \\
\hline$E_{g}-E_{H}$ & 0.267 & $\mathrm{eV}$ & M \\
\hline$N_{A}($ emitter $)$ & $2 \times 10^{18}$ & $\mathrm{~cm}^{-3}$ & M \\
\hline$E_{A}($ emitter $)$ & 0.036 & $\mathrm{eV}$ & $\mathrm{L}$ \\
\hline$N_{D}$ (base) & $2 \times 10^{17}$ & $\mathrm{~cm}^{-3}$ & M \\
\hline$E_{D}$ (base) & 0.006 & $\mathrm{eV}$ & $\mathrm{L}$ \\
\hline$N_{D}(\mathrm{QD}$ doping $)$ & $3.75 \times 10^{15}$ & $\mathrm{~cm}^{-3}$ & M \\
\hline$N_{I B}$ (QD density) & $1 \times 10^{16}$ & $\mathrm{~cm}^{-3}$ & M \\
\hline$\Delta E_{g, \text { base }}$ & 0.05312 & $\mathrm{eV}$ & $\mathrm{F}(I-V$ at $20 \mathrm{~K})$ \\
\hline $\mathscr{E}($ test cell $)$ & $70 \times 10^{3}$ & $\mathrm{~V} \mathrm{~cm}^{-1}$ & $\mathrm{~F}(\mathrm{PC} 1 \mathrm{D})$ \\
\hline$\tau_{n}($ test cell emitter $)$ & $6.74 \times 10^{-10}$ & $\mathbf{S}$ & \\
\hline$E_{\tau n}($ test cell emitter $)$ & $\mathbf{0}$ & eV & \\
\hline$\tau_{p}$ (base) & $6.744 \times 10^{-8}$ & $\mathrm{~s}$ & $\mathrm{~F}(I-V$ at $298 \mathrm{~K})$ \\
\hline$E_{\tau p}$ (base) & 0 & eV & \\
\hline$\tau_{s c}($ test cell $)$ & $4.478 \times 10^{-9}$ & $\mathrm{~s}$ & $\mathrm{~F}(I-V$ at $298 \mathrm{~K})$ \\
\hline$E_{\tau s c}($ test cell $)$ & 0 & $\mathrm{eV}$ & \\
\hline$\tau_{n}($ IB cell emitter $)$ & $2.39 \times 10^{-11}$ & $\mathbf{S}$ & \\
\hline$E_{\tau n}($ IB cell emitter $)$ & 0.0147 & eV & \\
\hline$\tau_{p}(\mathrm{IB}$ cell, $\mathrm{QD})$ & $2.39 \times 10^{-9}$ & $\mathrm{~s}$ & $\mathrm{~F}(I-V$ at $298 \mathrm{~K})$ \\
\hline$E_{\tau p}(\mathrm{IB}$ cell, $\mathrm{QD})$ & 0.0147 & $\mathrm{eV}$ & $\mathrm{F}(I-V$ at 150 and $77 \mathrm{~K})$ \\
\hline$\tau_{n}(\mathrm{IB}$ cell, $\mathrm{QD})$ & $1.69 \times 10^{-12}$ & $\mathrm{~s}$ & $\mathrm{~F}(I-V$ at $298 \mathrm{~K})$ \\
\hline$E_{\tau n}(\mathrm{IB}$ cell, QD) & 0.0147 & eV & \\
\hline$\tau_{s c I V}(\mathrm{IB}$ cell, QD) & $0.93 \times 10^{-9}$ & $\mathrm{~s}$ & $\mathrm{~F}(I-V$ at $298 \mathrm{~K})$ \\
\hline$E_{\tau s c I V}(\mathrm{IB}$ cell, QD) & 0.0531 & $\mathrm{eV}$ & $\mathrm{F}(I-V$ at $150 \mathrm{~K})$ \\
\hline$\tau_{s c C V}(\mathrm{IB}$ cell, $\mathrm{QD})$ & $0.93 \times 10^{-9}$ & $\mathbf{S}$ & \\
\hline$E_{\tau s c C V}(\mathrm{IB}$ cell, $\mathrm{QD})$ & 0.0531 & $\mathrm{eV}$ & \\
\hline $1+n_{r}^{2}$ & 14 & & $\mathrm{~L}$ \\
\hline$a_{\mathrm{CV}}$ & 1 & & $\mathrm{~L}$ \\
\hline$a_{\mathrm{IV}}$ & 0.0152 & & F (Figure 8) \\
\hline$a_{\mathrm{CI}}$ & 0.5 & & \\
\hline$\Delta E_{c}$ & 0.00949 & $\mathrm{eV}$ & F (Figure 8) \\
\hline$\Delta E_{v}$ & 0 & $\mathrm{eV}$ & \\
\hline $\mathscr{E}($ IB cell) & $5 \times 10^{3}$ & $\mathrm{~V} \mathrm{~cm}^{-1}$ & $\mathrm{~F}(\mathrm{PC} 1 \mathrm{D})$ \\
\hline
\end{tabular}

\section{A. Variable injection CB-VB non-radiative recombination current}

The region contains the QDs and a slight amount of donors introduced through a delta doping process meant to half-fill the QD levels. Most of the electrons introduced by the donors fall in the QD levels, but some of them are pumped in the dark by the thermal excitations and reach the $\mathrm{CB}$. In reality, those in the $\mathrm{CB}$ are regulated by the $\mathrm{CB} \mathrm{QFL}$ position. In the first instance we assume that this QFL is the same for the IB (at room temperature, this is the case for most operating conditions). Due to the large separation of the IB from the $\mathrm{CB}$, we can treat the QDs as "poorly ionized donors" in this region, but this effect has already been considered in our analysis [e.g., in Eq. (1)]. Charge neutrality is ensured by the donor impurities, which are almost fully ionized in all conditions, and the electrons in the IB and CB. When the cell is in high injection, the holes in the VB also enter into the balance.

Before this happens, the recombination current is ruled by Eqs. (2) and (3). Because the doping is very weak $\left(\sim 5 \times 10^{15} \mathrm{~cm}^{-3}\right)$ and not all the electrons are in the $\mathrm{CB}$ (many are in the IB), the majority carriers are very few, and consequently the minority carriers are very abundant, leading to a very strong recombination (as compared with the test cell with a more doped base). However, as shown in Sec. VI, this is not yet the dominant component.

Beyond a given voltage, the cell achieves high injection. The transition from low to high injection is mathematically involved (it cannot be solved with an explicit equation), but at very high injection, when electron and hole densities are equal and much higher than the doping and the whole region becomes intrinsic ( $n \cong p$ ), the equation becomes rather simple again (Ref. 21, p. 48). It is

$$
\begin{aligned}
& J_{h i} \cong K_{h i} \exp \left(\left(e V-E_{g}\right) / 2 k T\right), \\
& K_{h i} \cong \frac{e \sqrt{N_{c} N_{v}} W}{\tau_{p}+\tau_{n}} .
\end{aligned}
$$

Note that the voltage dependence is the same as that of the space-charge zone current. We have considered the diode as short in this case. We have already discussed the small relevance of a more exact consideration. In the high injection regime, the space-charge recombination must be discarded because the entire QD region is operating under the high recombination conditions of the space-charge zone.

The transition from low to high injection is again treated in an oversimplified way. The low injection current prevails at low voltage, and the high injection current conditions prevail at high voltage. We simply write the actual current as the inverse of the sum of the inverses of the low injection and high injection cases, and we call it $J_{l h i}$.

\section{B. IB-VB non-radiative recombination current}

The analysis of this important current (actually the most important at room temperature) requires that we revisit SRH recombination. ${ }^{25,27,28}$ Let us assume there is a trap of density $N_{t}$. Although these traps are common even in non-structured semiconductors, the existence of different localized traps associated with strain relaxation in InAs/GaAs QDs has been reported in the literature. ${ }^{29}$ For simplicity, let us assume that this trap is characterized by a deep level $E_{t}$ situated more or less midway between the IB and the $\mathrm{VB}$, and let the factor $f$ be the occupation fraction of the trap by electrons. Four mechanisms must be considered: the emission of holes by the trap level at a rate of $e_{p} N_{t}(1-f)$, the capture of holes by the trap level at a rate of $v_{t h} \sigma_{p} p N_{t} f$, the emission of electrons to the IB by the trap level at a rate of $e_{I B} N_{t} f$, and the capture of IB electrons by the trap at a rate of $c_{I B} n_{I B} N_{t}(1-f)$. The factors $e_{p}, v_{t h} \sigma_{p}, e_{I B}$, and $c_{I B}$ are the dynamic coefficients of the four processes. The hole capture mechanism is decomposed into a capture section and a thermal velocity as usual in the bands, but in the case of the IB electrons, no further assumption has been made about their capture by the trap. Under thermal equilibrium, $f$ is the Fermi factor corresponding to the trap energy level and $p=p_{e q}, n_{I B}=n_{I B e q}$. Because in thermal equilibrium the detailed balance requires that the capture and emission processes be the same, the emission coefficients can be calculated as a function of the capture 
coefficients. Once this is done, a steady state equation can be established that states that the net incoming flux of electrons to the trap from the IB must equal the outgoing flux toward the $\mathrm{VB}$, and this rate is the recombination rate. The equality established allows the calculation of $f$ under non-equilibrium conditions. Once $f$ is obtained, the recombination is calculated, and the following equation is obtained:

$$
r=\frac{n_{I B} p-n_{I B e q} p_{e q}}{\tau_{p}\left[n_{I B}+n_{I B e q} \exp \left(\frac{E_{t}-E_{F}}{k T}\right)\right]+\tau_{I B}\left[p+N_{v} \exp \left(\frac{E_{v}-E_{t}}{k T}\right)\right]},
$$

where $1 / \tau_{p}=v_{t h} \sigma_{p} N_{t}$, as usual, and $1 / \tau_{I B}=c_{I B} N_{t}$ of unknown temperature dependence. This expression resembles the ordinary SRH recombination formula, but the equilibrium Fermi-level position, absent in the ordinary SRH formula, is present here.

\section{Neutral zone}

In the neutral zone, for $N_{I B}>N_{D}, n_{I B e q}=N_{D}$, and in low injection $n_{I B}=n_{I B e q}=N_{D}$. In equilibrium, the Fermi level is located at

$$
\frac{N_{I B}-n_{I B}}{N_{D}} \exp \left(\frac{E_{F}-E_{I B}}{k T}\right)=1,
$$

where $n_{I B}=N_{D}$ must be used for the determination of the Fermi level. Therefore,

$$
n_{I B e q} p_{e q}=\left(N_{I B}-N_{D}\right) N_{v} \exp \left(\frac{E_{v}-E_{I B}}{k T}\right) .
$$

The role of $N_{c}$ in ordinary semiconductors is taken here by $N_{I B}-N_{D}$, and the bandgap is replaced by the so-called high sub-bandgap $E_{H}=E_{I B}-E_{v}$.

Taking this into account, in neutral zones,

$$
r=\frac{n_{I B} p-n_{I B e q} p_{e q}}{\tau_{p}\left[n_{I B}+\left(N_{I B}-n_{I B e q}\right) \exp \left(\frac{E_{t}-E_{I B}}{k T}\right)\right]+\tau_{I B}\left[p+N_{v} \exp \left(\frac{E_{v}-E_{t}}{k T}\right)\right]} \cong \frac{p-p_{e q}}{\tau_{p}}
$$

We have assumed that in this case $n_{I B}=n_{I B e q}=N_{D}$. Note that the approximate expression to the right requires that the term $\tau_{p} n_{I B}$ be the largest in the denominator. This will usually be true (at least as long as $p$ is a minority carrier) if $\tau_{I B}$ is not extremely long, reflecting the fact that the transitions between the trap and the IB are negligible. If the latter is not the case, the recombination is regulated by the ordinary hole lifetime (provided that the trap level is the same as the one for the CB-IB recombination). Actually, this expression is also valid if $n_{I B}$ is above the equilibrium value, entering in high injection for the IB electrons.

This equation is formally identical to the one that holds for $\mathrm{CB}$ and $\mathrm{VB}$ electrons and holes, changing $N_{c}$ into $N_{I B}-N_{D}$ and $E_{g}$ into $E_{H}$ so that the recombination current is

$$
\begin{aligned}
J_{I V} & \cong K_{01 I V} \exp \left(\left(e V-E_{H}\right) / k T\right), \\
K_{01 I V} & =e N_{v}\left(\frac{N_{I B}-N_{D}}{N_{D}}\right) \frac{W}{\tau_{p}},
\end{aligned}
$$

in which we have abandoned the complexities associated with the long diode. Notice that this current is rather high, essentially because the applicable bandgap $E_{H}$ is smaller.

With symmetric arguments, we can calculate the CB-IB non-radiative recombination current,

$$
\begin{aligned}
J_{C I} & \cong K_{01 C I} \exp \left(\left(e V-E_{g}+E_{H}\right) / k T\right), \\
K_{01 C I} & =e N_{c}\left(\frac{N_{D}}{N_{I B}-N_{D}}\right) \frac{W}{\tau_{n}} .
\end{aligned}
$$

It must be noted that $\tau_{n}$ now corresponds to a trap level situated between the IB and the CB that in the general case will be different from the one ruling the $\mathrm{CB}-\mathrm{VB}$ recombination.

\section{Space-charge zone}

Notice that Eq. (7) is also valid in the space-charge zone. Furthermore, the $n_{\text {IBeq }} p_{e q}$ product in Eq. (8) is valid as well. Because the Fermi level (calculated in the neutral zone) is constant in the space-charge zone with the bands bending upward as the concentration of holes increases toward the p-emitter, the IB electrons decrease in the same proportion. Equation (9) is also applicable to this case, although now $n_{I B e q} \ll N_{D}$. This equation is formally identical to the one holding for $\mathrm{CB}$ electrons and VB holes, changing $N_{C}$ to $N_{I B}, \tau_{n}$ to $\tau_{I B}$, and $E_{g}$ to $E_{H}$ so that the recombination current is

$$
\begin{aligned}
J_{s c z I V} \cong K_{02 I V} \exp \left(\left(e V-E_{H}\right) / 2 k T\right), \\
K_{02 I V} \cong \frac{e \sqrt{N_{I B} N_{v}}}{2 \sqrt{\tau_{p} \tau_{I B}}} \frac{k T}{e \mathrm{E}} .
\end{aligned}
$$

Again here, the current might be high because the applicable sub-bandgap $E_{H}$ is smaller.

No space-charge zone current is considered for the CBIB recombination because the neutral zone current is already considered to be very large and, due to the low value of the sub-bandgap, it might be blurred by the radiative recombination. In addition, very little experimental information is available on this transition.

\section{THE RADIATIVE CURRENTS}

The radiative recombination is given by the van Roosbroeck-Shockley formula, ${ }^{30}$ 


$$
\begin{aligned}
J_{\text {rad } C V}= & \frac{e 2 \pi\left(1+n_{r}^{2}\right)}{h^{3} c^{2}} \int_{E_{g}}^{\infty} \frac{a E^{2} d E}{\exp \left(\left(E-E_{F}\right) / k T\right)-1} \\
\cong & \frac{e 2 \pi\left(1+n_{r}^{2}\right)}{h^{3} c^{2}} \int_{E_{g}}^{\infty} a E^{2} \exp \left(\left(E-E_{F}\right) / k T\right) d E \\
= & \frac{e 2 \pi a\left(1+n_{r}^{2}\right)}{h^{3} c^{2}}(k T)^{3}\left(2+\frac{2 E_{G}}{k T}+\left(\frac{E_{G}}{k T}\right)^{2}\right) \\
& \times \exp \left(\frac{e V-E_{G}}{k T}\right) .
\end{aligned}
$$

The recombination is associated with the emission of photons, but many are reabsorbed, and only those escaping the semiconductor contribute to the recombination. In this calculation, it is assumed that the VB-CB QFL splitting is constant. This is reasonably accurate for short diodes with no back recombination. The term $\left(1+n_{r}^{2}\right)$, in which $n_{r}$ denotes the semiconductor index of refraction, refers to the fact that the photons are emitted through the front face (term 1) and also through the back face (term $n_{r}$ ) toward the thick semiconductor substrate, where they are absorbed (mainly by free carrier absorption), and they do not return. The factor $a$ is the absorbance that, according to Kirchhoff's law, equals the emittance (fulfilled if the QFL splitting is constant ${ }^{31}$ ). For the CV-VB transitions it has been set to one, independent of the photon energy (the semiconductor is thick enough).

The pre-exponential factor of the analytic expression can be termed $K_{\text {radCV }}$ and shows temperature dependence approximately proportional to $T$, in contrast to $K_{01}$, which is approximately proportional to $T^{3}$. It is represented in Fig. 5 . It is visible that the radiative recombination increases with the temperature much more slowly. At room temperature it is smaller than the emitter and base non-radiative currents, but at low temperature it dominates, except in the absence of the Arrhenius energy for the capture cross-section of the non-radiative process.

Similar expressions must be considered for the IB-VB and $\mathrm{CV}$-IB transitions. In the ideal case, the absorbance is selective; i.e., the integral must be extended from $E_{H}$ to $E_{g}$ for the IB-VB emissions and from $E_{g}-E_{H}$ and $E_{H}$ for the CB-IB transition. However, its value can be well below unity because the absorption in these bands may be partial, even very small. Here it is useful to remember that the integration between two finite boundaries is the subtraction of the integrals from each boundary to infinity.

\section{THE IB-REGION COMBINED CURRENT}

In the preceding section, we attempt to examine, one by one, the different components of the recombination current, with special emphasis on their low temperature behavior. Our main finding is that the dominant recombination term, at least at room temperature, is associated with the SRH recombination but is modified with respect to the ordinary cells in the sense that transitions between the IB and impurity levels are not negligible. This last condition is found to be true, as discussed in Sec. VI.
For the rest it is found that the normally used formulas are applicable to low temperature operation, assuming that there is an activation energy for the recombination capture section, as proposed by Henry and Lang. ${ }^{23}$ Furthermore, at low temperature the diffusion length tends to increase due to the increase of the mobility so that the diodes become short, therefore simplifying the presentations of the formulas and, to some extent, the calculations. Additionally, the low temperature Auger recombination holding in the emitter has not been described in the paper for the sake of brevity. This might affect somewhat the test (ordinary) cell behavior, but certainly not the IB solar cell. Finally, it is to be noted that the dominant role is taken by the non-radiative recombination at low temperature. All these aspects and statements are further discussed and substantiated in Sec. VI.

The task in this section is to solve the circuit in Fig. 1 in the dark (no current sources). In an IB solar cell, the current is derived along two branches: one across diodes D2 and D3, called $J_{Q D}$, and one across diode D1. Let us consider the first. The voltages across each diode, which in reality represent the position of the VB and CB QFLs with respect the IB QFL, are

$$
\begin{aligned}
V_{V I}= & \frac{E_{H}}{e} \\
& +\frac{2 k T}{e} \log \left(\frac{\sqrt{K_{s c z I V}^{2}+4 J_{Q D}\left(K_{01 I V}+K_{\text {radIV }}\right)}-K_{s c z I V}}{\left(K_{01 I V}+K_{\text {radIV }}\right)}\right) \\
& V_{I C}=\frac{E_{g}-E_{H}}{e}+\frac{k T}{e} \log \left(\frac{J_{Q D}}{\left(K_{01 C I}+K_{\text {radCI }}\right)}\right)
\end{aligned}
$$

To obtain the first equation, consider that $J_{Q D}$ across D2 is the sum of the radiative and non-radiative recombination currents along this path proportional to $\exp \left(\left(e V_{I V}-E_{H}\right) / k T\right)$ and the space-charge zone current that is proportional to $\exp \left(\left(e V_{C I}-E_{H}\right) / 2 k T\right)$. We can take the latter as the variable and solve a 2 nd order ordinary equation. Equation (15) is obvious and also considers the radiative recombination in this sub-bandgap.

The sum of these two voltages is the applied voltage $V$ and provides a $V\left(J_{Q D}\right)$ relationship that is inverted numerically to obtain $J_{Q D}(V)$. Thus, the total current in the QD zone is

$$
J_{I B}(V)=J_{Q D}(V)+J_{l h i}(V)+J_{s c z}(V)+J_{\operatorname{rad} C V}(V) .
$$

\section{DISCUSSION}

Figure 6 shows the measured ${ }^{32}$ values of the $J_{\mathrm{L}}-V_{\mathrm{OC}}$ curves for the test solar cell and the IB solar cell at different temperatures, together with the fitted curves. The parameters used for the calculations in our model are in Table I. As indicated in the table, these parameters are from the literature, from measurements of the sample under study, or from fitting. Some parameters without any indication of origin, lettered in bold, are taken as what the authors consider reasonable. 

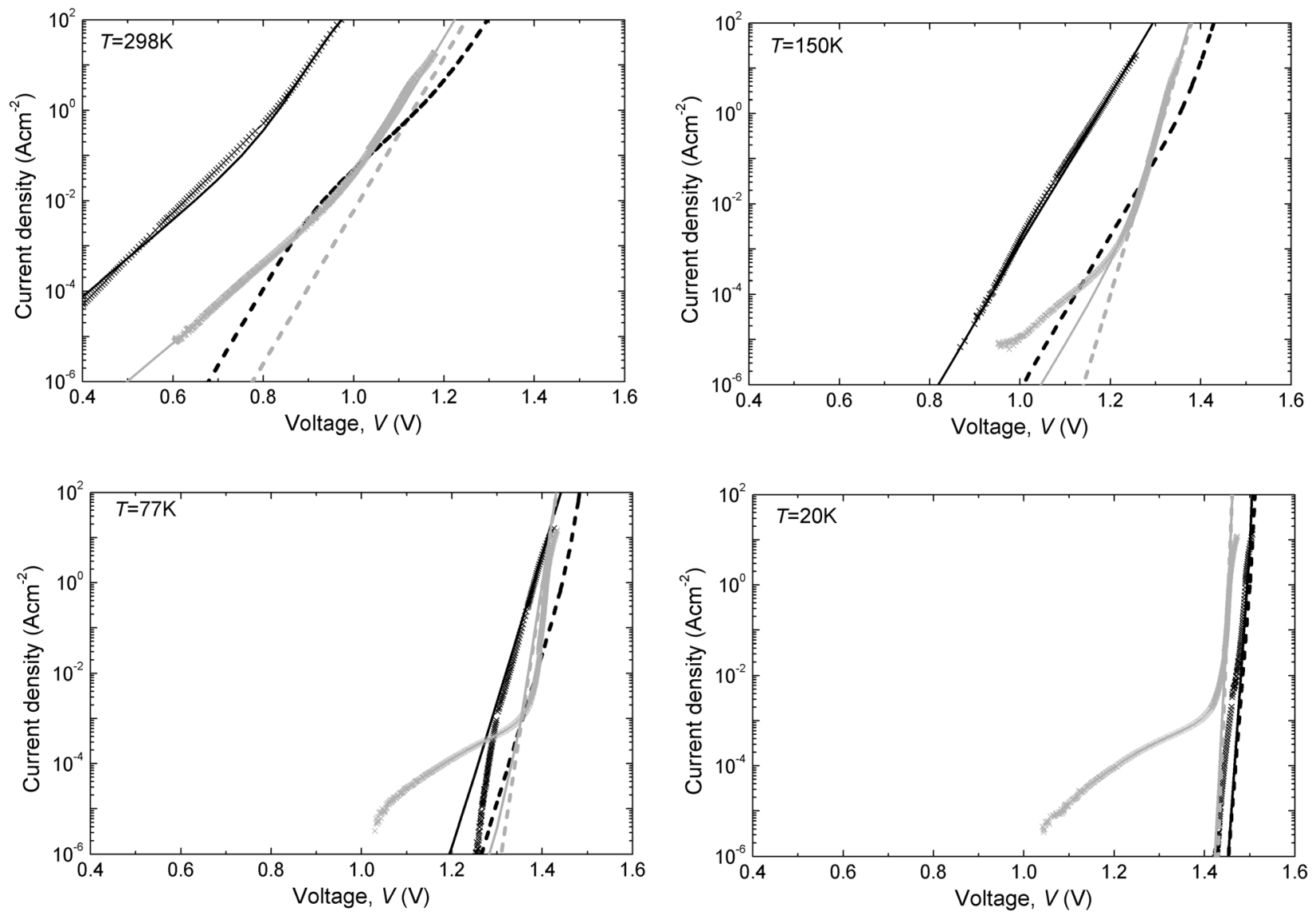

FIG. 6. Calculated (solid and dashed lines) and measured (crosses) $J_{\mathrm{L}}-V_{\mathrm{OC}}$ curves at several temperatures for the test cell (gray) and the IB cell (black). The dashed lines refer to purely radiative calculations, neglecting all non-radiative components.

In the same figures, the result of considering only radiative recombination in the model is also presented. The dashed light gray lines represent the test cell radiative recombination current, which has a slope $e / k T$ that obviously becomes steeper as the temperature decreases. The dashed black lines represent the IB cell radiative recombination current. The latter theoretically present two zones of slope $e / k T$ (not visible within the span of the drawings) connected by a region of lower slope. The two former vary approximately as $\exp \left(\left(e V-E_{H}\right) / k T\right)$ and $\exp \left(\left(e V-E_{g}\right) / k T\right)$, with the higher current associated with the IB-VB transitions. When the voltage increases, $V_{I C}$ increases, and the weight of $\exp \left(\left(\mathrm{eV}-E_{H}\right) / k T\right)$ decreases until it becomes negligible. The region of lower slope appears at the onset of $V_{I C} \neq 0$. This means that the recombination vestiges due to the IB-VB luminescence, which are a cause of reduced efficiency, disappear at the high voltages achieved under high concentration operation. This fact was exposed in Ref. 33. This behavior is also found here for recombination that is dominantly non-radiative.

\section{A. The test cell fitting}

The recombination current in the test solar cell is simulated as the sum $J_{\text {em,test }}+J_{\text {base }}+J_{\text {scz,test }}+J_{\text {radCV }}$. There is an important experimental fact that must be taken into account; namely, the test cell shows a voltage (for a given current) that is lower than that of the IB cell at $20 \mathrm{~K}$ (see Fig. 6). This seems to reveal a lower bandgap in the test cell than in the IB cell. As the only difference is the addition of an n-type base region to the former that is rather thick $(3.1 \mu \mathrm{m})$, we deduce that the n-base suffers bandgap shrinkage, perhaps due to doping. This is confirmed by the appearance of a red-shift in the electroluminescence peak at very low temperature that is absent in the IB solar cell. This shift does not appear in photoluminescence (confirming that there is no $p$-emitter shrinkage) or at higher temperatures that might be due to optical bandgap widening due to heavy population of the bottom by electrons from the dopants, as reported by some authors. $^{22}$

The amount of this shrinkage is on the order of $0.05 \mathrm{eV}$, as revealed by the luminescence data and also by the difference of the voltages. At $1.45 \mathrm{~V}$ at $20 \mathrm{~K}$, the main contribution is the radiative term $J_{\mathrm{radCV}}$, as is made clear in Fig. 5, so that the bandgap shrinkage can be fitted. Taking into account that $a=1$ and $\left(1+n_{r}^{2}\right)=14, \Delta E_{\mathrm{g}, \text { base }}=0.05312 \mathrm{eV}$. Once the base bandgap has been shrunk, the dominant current at $0.6 \mathrm{~V}$ and $298 \mathrm{~K}$ is $J_{\text {scz,test }}$. This allows us to fit $\tau_{\text {test cell }}=2 \sqrt{\tau_{p} \tau_{n}}=4.478 \times 10^{-9} \mathrm{~s}$. Finally, we fit the $298 \mathrm{~K}$ current at $1.18 \mathrm{~V}$ with a value of $\tau_{p}=6.744 \times 10^{-8} \mathrm{~s}$ in the base. The calculated value for $\tau_{n}$ is $\tau_{n}=7.43$ $\times 10^{-11} \mathrm{~s}$. As a matter of fact, we ignore the relative contribution of the base and emitter currents, but we assume that the lifetime in the emitter is 100 times smaller than $\tau_{p}$ in the base, because the doping is ten times higher: the $\mathrm{Au}-$ ger lifetime is inversely proportional to the square of the 
doping (although this rule applies to carriers of the same polarity, which is not the case).

As for the cause for the bandgap shrinkage, we recall that the fine structure in the $\mathrm{CB}$ bottom is very much affected by dopants and maybe other shallow impurities, depending on the sample. This makes the value of $E_{g}$ given in Eq. (5) uncertain, and that of $N_{c}$ in the literature should be taken as strictly valid only for "perfect" non-degenerate semiconductors.

At high voltage, the test cell fitting is rather good, but this is not true at low current, where some kind of leakage seems to occur at low temperatures that cannot be explained by recombination processes. Maybe it is specific to the studied device, rather than a general feature. For the test cell, the current observed corresponds to the radiative recombination as soon as we reduce the temperature. This prevents us from determining the Arrhenius term of the lifetimes that influences their temperature behavior. An exception is the measurement at $77 \mathrm{~K}$, where the voltage is beyond that expected from the radiative recombination with the fitted bandgap shrinkage.

The current components under several voltage and temperature conditions are presented in Table II. The distribution between emitter and base current is arbitrary, but the emitter current becomes very small at low temperatures, and its dependence on the voltage is indistinguishable from that of the base at room temperature. Any distribution is equally valid for fitting purposes.

In summarizing our opinion on this fitting, which is intended to explain the low voltage at low temperature, we must declare that we are uneasy with the large value of $\tau_{p}$ and its big difference from $\tau_{n}$. Maybe our assumption that at $20 \mathrm{~K}$ all the current at high voltage is radiative is not completely right, or maybe the bandgap shrinkage is variable with the temperature (although we do not foresee any mechanism for it). More experiments might be necessary, but this paper is not specifically involved with investigating the low temperature behavior of an ordinary solar cell.

\section{B. The intermediate band solar cell fitting}

The IB cell current is $J_{e m}+J_{I B}$ (that is, $\left.J_{e m}+J_{Q D}+J_{l h i}+J_{s c z}+J_{r a d C V}\right)$ based on Eq. (16). In turn, $J_{Q D}=\left(K_{01 I V}+K_{\text {radIV }}\right) \exp \left(e V_{V I} / k T\right)+K_{02 I V} \exp \left(e V_{V I} / 2 k T\right)$, where $K_{\text {radlV }}$ is similar to $K_{\text {radCV }}$, using $E_{H}$ as the bandgap. $V_{V I}=V-V_{I C}$ is calculated with Eq. (15). In other words, we have three variables- $J_{Q D}, V_{V I}$, and $V_{I C}$ - to be solved for

TABLE II. Current components of the test cell for various operational conditions.

\begin{tabular}{lccc}
\hline \hline & $\mathrm{V}=1.18054 \mathrm{~V}$ & $\mathrm{~V}=0.603338 \mathrm{~V}$ & $\mathrm{~V}=1.45036 \mathrm{~V}$ \\
$\mathrm{~T}=298 \mathrm{~K}$ & $\mathrm{~T}=298 \mathrm{~K}$ & $\mathrm{~T}=20 \mathrm{~K}$ \\
\hline$J_{\text {em,test }}$ & 3.27574 & $5.67207 \times 10^{-10}$ & $3.16071 \times 10^{-13}$ \\
$J_{\text {base }}$ & 8.41015 & $1.45625 \times 10^{-9}$ & 0.00371 \\
$J_{\text {scz,test }}$ & 0.58420 & $7.68732 \times 10^{-6}$ & $1.04117 \times 10^{-4}$ \\
$J_{\text {radCV }}$ & 6.86309 & $1.18837 \times 10^{-9}$ & 0.17660 \\
Calculated current & 19.1332 & $7.69053 \times 10^{-6}$ & 0.18048 \\
Measured current & 19.1335 & $7.69 \times 10^{-6}$ & 0.18088 \\
\hline \hline
\end{tabular}

each $V$ with the preceding equation and Eqs. (14) and (15). In order to keep the nomenclature simple, we can express the equation above as $J_{Q D}=J_{01 I V}+J_{\text {radIV }}+J_{02 I V}$. Once the fitting is done, all these values can be calculated; they are given in Table III for several operating conditions.

$J_{Q D}$ is a very important source of recombination in the IB cell because the bandgaps associated are small. The larger of them, $E_{H}$, is indeed smaller than $E_{g}$. At room temperature $(298 \mathrm{~K})$ and $0.902 \mathrm{~V}$, the dominant current is $J_{01 I V}$, but its voltage dependence cannot be distinguished from that of the emitter of even the CB-VB recombination. We may keep the same arbitrary assumption as with the test cell-that is, keep that emitter lifetime 100 times smaller than $\tau_{p}$ in the IB region. With this assumption, $\tau_{p}=2.39 \times 10^{-9} \mathrm{~s}$, and the emitter electron lifetime is 100 times smaller. The latter value is lettered in bold in Table I to stress that the value selected is in fact arbitrarily selected, although it is of a reasonable value.

At $0.502 \mathrm{~V}$, the same uncertainty occurs for the dominant terms $J_{s c z}$ and $J_{02 I V}$, again with the same voltage dependence. Arbitrarily, we have set them equal, although $J_{02 I V}$ is dominant due to its smaller bandgap (again, the lifetime leading to the non-dominant term is lettered in bold in Table I). Once this is done, $\tau_{s c l V}=2 \sqrt{\tau_{p} \tau_{I B}}$ can be fitted as $\tau_{s c I V}=0.93 \times 10^{-9} \mathrm{~s}$. $\tau_{I B}$ is then calculated as $\tau_{I B}=0.905 \times 10^{-10} \mathrm{~s}$, which is small enough to permit the assumption that the recombination in the neutral zone is ruled by the ordinary base hole lifetime, as adopted in Sec. III.

The fitting at $298 \mathrm{~K}$ is reasonably good, as seen in Fig. 6 . It must be noted that for simplicity, we have considered the IB as a single level in this paper, even though we know it is formed by several levels ${ }^{9,12,34-36}$; this might explain the imperfect fitting in the medium voltage range, which is much better in Ref. 36. In Fig. 7 we show the modeled curve and compare it with its components $J_{02 I V}+J_{s c z}$ and $J_{01 I V}$. The onset of a lower slope behavior appears at the highest voltages, representing the onset of $V_{I C} \neq 0$. This onset is also visible in the measured curve. It is ruled by $\tau_{n}$, which now refers to the CB-IB recombination and has been set at

TABLE III. Current components of the IB cell for various operational conditions.

\begin{tabular}{lcccc}
\hline \multirow{2}{*}{$\begin{array}{l}\text { Current } \\
\left(\mathrm{A} \mathrm{cm}{ }^{-2}\right)\end{array}$} & $\begin{array}{c}\mathrm{V}=0.90204 \mathrm{~V}, \mathrm{~V}=0.50230 \mathrm{~V}, \mathrm{~V}=1.19956 \mathrm{~V}, \mathrm{~V}=1.50018, \\
\mathrm{~T}=298 \mathrm{~K}\end{array}$ & $\mathrm{~T}=298 \mathrm{~K}$ & $\mathrm{~T}=150 \mathrm{~K}$ & $\mathrm{~T}=20 \mathrm{~K}$ \\
\hline$J_{\text {em }}$ & 0.00102 & $1.77 \times 10^{-10}$ & $1.84 \times 10^{-5}$ & 0.01146 \\
$J_{\text {IB }}$ & 10.3384 & $5.60 \times 10^{-4}$ & 2.61397 & 7.75146 \\
Sum (total $J)$ & 10.3394 & $5.60 \times 10^{-4}$ & 2.61399 & 7.76292 \\
Measured & 10.3658 & $5.61 \times 10^{-4}$ & 2.69872 & 7.18752 \\
$J_{\text {lhi }}$ & 0.26337 & $5.98 \times 10^{-8}$ & 0.06219 & $2.54 \times 10^{-4}$ \\
$J_{\text {scz }}$ & 0.06098 & $2.54 \times 10^{-5}$ & $4.21 \times 10^{-4}$ & $8.74 \times 10^{-16}$ \\
$J_{\text {radCV }}$ & $2.60 \times 10^{-5}$ & $4.52 \times 10^{-12}$ & $3.49 \times 10^{-6}$ & 6.78041 \\
$J_{\mathrm{QD}}$ & 10.0140 & $5.35 \times 10^{-4}$ & 2.55136 & 0.97080 \\
Sum $\left(J_{\text {IB }}\right)$ & 10.3384 & $5.60 \times 10^{-4}$ & 2.61397 & 7.75146 \\
$V_{\text {IC }}(\mathrm{V})$ & 0.00130 & $7.13 \times 10^{-8}$ & 0.10177 & 0.25305 \\
$J_{01 I V}$ & 8.87093 & $1.60 \times 10^{-6}$ & 2.49649 & 0.03004 \\
$J_{\text {radIV }}$ & 0.00574 & $1.05 \times 10^{-9}$ & 0.00620 & 0.94075 \\
$J_{02 I V}$ & 1.24736 & $5.33 \times 10^{-4}$ & 0.04868 & $4.39 \times 10^{-14}$ \\
Sum $\left(J_{\mathrm{QD}}\right)$ & 10.0140 & $5.35 \times 10^{-4}$ & 2.55136 & 0.97080 \\
\hline \hline
\end{tabular}




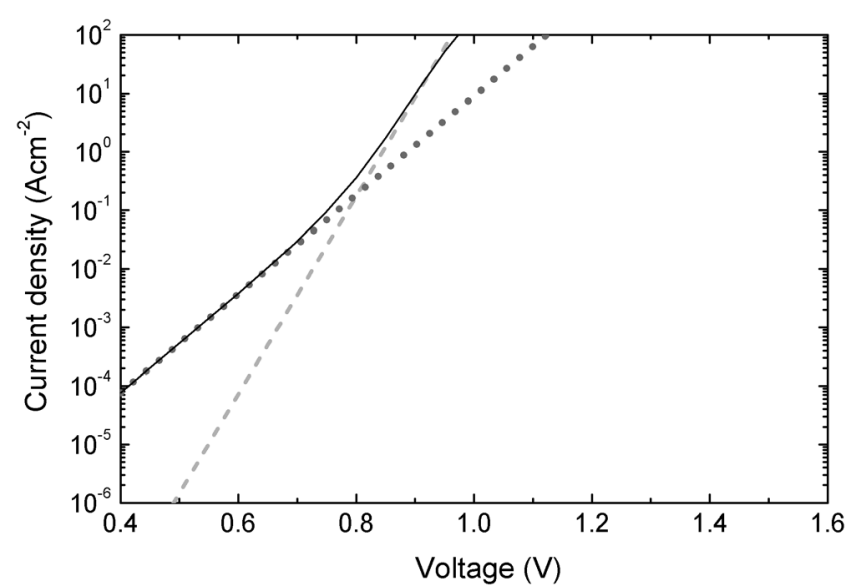

FIG. 7. Modeled $J_{\mathrm{L}}-V_{\mathrm{OC}}$ curve (solid black line) at $298 \mathrm{~K}$ and $J_{02 I V}+J_{s c z}$ (dotted dark gray line) and $J_{01 I V}$ (dashed light gray line) components.

$\tau_{n}=1.69 \times 10^{-12} \mathrm{~s}$ in order to obtain a good fitting at $V=0.98152 \mathrm{~V}$. At this voltage, $V_{I C}=0.01304 \mathrm{~V}$. With regard to this fitting, the terms $K_{01 C I}$ (the one affected by $\tau_{n}$ ) and $K_{r a d C I}$ are indistinguishable when determining $V_{I C}$, as is evident in Eq. (15). The parameter $a_{C V}$ has been set arbitrarily at $a_{C I}=0.5$ and is lettered in bold in Table I.

Some iteration is necessary in order to obtain simultaneously the fittings at the three voltages $(0.902,0.502$, and $0.982 \mathrm{~V})$ described above.

Now let us consider the data at $20 \mathrm{~K} .{ }^{19}$ In this range, radiative recombination dominates, as shown in Fig. 8, and the non-radiative recombination is believed to be rather small. Our model confirms this situation if the Arrhenius factors of the lifetimes are large enough, as shown in Fig. 4. In this respect, Table III shows that the most important term at $1.5002 \mathrm{~V}$ is $J_{\text {radCV }}$, followed by $J_{\text {radIV }}$. This order is reversed when we set a lower bias, as can be seen in Fig. 8, in which the total current injected is $0.08 \mathrm{~A} / \mathrm{cm}^{2}$.

At $20 \mathrm{~K}$ the logarithmic slope of $J_{\text {radIV }}$ is found to be $e / 2 k T$. Let us call $V_{0.08}$ the voltage at which the cell yields $0.08 \mathrm{~A} \mathrm{~cm}^{-2}$ at $20 \mathrm{~K}$. As the ratio $J_{\text {radIV }} / J_{\text {radCV }}$ $=0.7 / 0.3$ and both currents must sum to $0.08 \mathrm{~A} \mathrm{~cm}^{-2}$, their

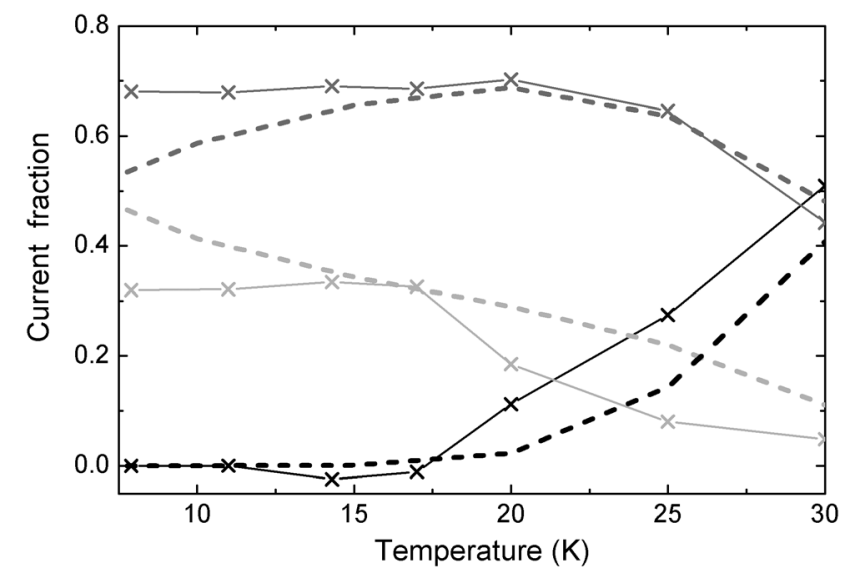

FIG. 8. Proportion of radiative and non-radiative recombination mechanisms at different temperatures for a current of $0.08 \mathrm{~A} \mathrm{~cm}^{-2}$. Light gray lines, $J_{\text {rad CV }}$; dark gray lines, $J_{\text {radIV }}$; black lines, non-radiative recombination current; solid lines with crosses, measurements; dashed lines, model. values must be $J_{\text {radCV }}=0.024 \mathrm{~A} \mathrm{~cm}^{-2}$ and $J_{\text {radIV }}$ $=0.056 \mathrm{~A} \mathrm{~cm}^{-2}$. The currents at $1.50018 \mathrm{~V}$ are, respectively, $0.024 \exp \left(e\left(1.50018-V_{0.08}\right) / 20 k\right) \mathrm{A} \mathrm{cm}^{-2}$ and $0.052 \exp$ $\left(e\left(1.50018-V_{0.08}\right) / 2 \cdot 20 k\right) \mathrm{A} \mathrm{cm}^{-2}$; their sum must be the experimentally measured $7.1875 \mathrm{~A} \mathrm{~cm}^{-2}$. This allows us to obtain $V_{0.08}=1.49045 \mathrm{~V}$, which should yield a calculated value of $0.08 \mathrm{~A} \mathrm{~cm}^{-2}$. With this voltage, we can calculate all the currents and adjust $J_{\mathrm{radCV}}=0.024 \mathrm{~A} \mathrm{~cm}^{-2}$ and $J_{\text {radIV }}=0.052 \mathrm{~A} \mathrm{~cm}^{-2}$ as desired. It is better to adjust $J_{\text {radCV }}=0.024 \mathrm{~A} \mathrm{~cm}^{-2}$ first. The parameter for fitting is a reduction $\Delta E_{c}=0.00949 \mathrm{eV}$ of the optical bandgap $E_{g}$ to be attributed to the wetting layer quantum well (appearing for QDs grown in the Stranski-Krastanov mode).

Actually, the optically observed wetting layer shrinkage is about $0.06 \mathrm{eV}$, but its density of states is weak, implying a small absorbance for the energies involved. As we are assuming an absorbance of unity, it is not surprising that the fitting value is much smaller (only $0.00949 \mathrm{eV}$ ).

Once the current $J_{\mathrm{radCV}}$ has been correctly fitted, we proceed to the fitting of $J_{\text {radIV }}$, this time by adjusting the VB-IB absorbance. The fitting is achieved for an absorbance $a_{I V}=0.0152$ for $1+n_{r}^{2}$ as in Table I. As a matter of fact, the absorbance for the VB-IB transitions has been measured for this specific cell, ${ }^{12}$ and it ranges between 0.005 for $0.9 \mathrm{eV}$ and 0.03 for $1.35 \mathrm{eV}$. We are considering a bandgap at room temperature $E_{H}=1.156 \mathrm{eV}$. The measured absorbance at this energy $^{12}$ is about 0.011 . The averaged value of 0.0152 seems to concur with these measurements. We might have set some shrinkage and simultaneously reduced the absorbance, but we have taken the simplest choice (no shrinkage). We also should have considered that $1+n_{r}^{2}$ at the IB-VB luminescence peak is somewhat smaller than the value in Table I. Taking this into account, $a_{I V}$ would have had a slightly increased value, but the change is not important.

The bandgap shrinkage has been applied only to the radiative currents, not to the non-radiative ones. This is debatable, but anyway, the Arrhenius factor that expresses the lifetime increase with the temperature such that attributing a gap shrinkage is (very approximately) the same as setting the $E_{\tau p}$, with the only difference being that the fitting lifetime is reduced in the first case to $\tau_{p, \text { shrink }}(300) \propto \tau_{p, \text { Arrh }}(300) \exp \left(-E_{\tau p} / 300 k\right)$. In other words, we may subtract the bandgap shrinkage from the Arrhenius factor only if we multiply the fitted lifetime by a factor $\exp \left(E_{\tau p} / 300 k\right)$, thus increasing it. In this way, the IB lifetimes might be closer to those obtained for the test cell.

Setting aside the last comment, measurements at $150 \mathrm{~K}$ or $77 \mathrm{~K}$ allow an estimate of the Arrhenius factors for some lifetimes. For the $150 \mathrm{~K}$ measurements, at low voltage $(0.89857 \mathrm{~V})$ the $J_{s c z I V}$ term is dominant. This allows us to fit $E_{\tau s c I V}=\left(E_{\tau p}+E_{\tau I B}\right) / 2=0.0531 \mathrm{eV}$, corresponding to the lifetime in Eq. (12) when, for simplicity, we keep $E_{\tau s c C V}=E_{\tau s c I V}$ (other combinations might also produce the fitting). At a higher voltage of $1.19956 \mathrm{~V}$, the dominant term is $J_{01 I V}$. This allows us to set $E_{\tau p}=0.0147 \mathrm{eV}$ when we set $E_{\tau p}=E_{\tau n}=E_{\tau, \mathrm{emm}}$ (other combinations also are possible). As usual, the energies corresponding to non-dominant terms are lettered in bold in Table I. From these values, we can deduce $E_{\tau I B}=0.0815 \mathrm{eV}$. When the $150 \mathrm{~K}$ curve is fitted accurately, the calculated curve lies slightly above the 
measured one at $77 \mathrm{~K}$, and with accurate fitting at $77 \mathrm{~K}$ the calculated curve is fractionally below. We have decided to set the $E_{\tau p}$ fitting as the arithmetic mean of both accurate values, that is, $E_{\tau p}=0.0147 \mathrm{eV}$; the fitting still looks very nice in Fig. 6.

The data in Fig. 8 deserve more attention. For further discussion, we present in Table IV the calculated breakdown of currents when the total current is set at $0.08 \mathrm{~A} \mathrm{~cm}^{-2}$ (the value in the experiments).

As is evident in Fig. 8, our model cannot explain the measured similarity of the IB-VB radiative recombination and the total non-radiative recombination in a wide range of temperatures. More details appear in Table IV. Only at $20 \mathrm{~K}$ is the proportion the experimental one (as results from our fitting). It is important to realize that our assumption that all the recombination is radiative at this temperature is only approximately confirmed by the model, with the current $J_{01 I V}$ still being perceptible. At higher temperatures, the radiative currents decrease and become insignificant.

The data in Table IV cast some additional light with respect to the data in Table III. In almost all cases, the main source of recombination is through the IB introduced by the QDs as revealed by the current $J_{Q D}$ (excepting the results at $20 \mathrm{~K}$ and high voltage, in which the radiative CB-VB recombination dominates). Thus it is inherent to their presence. Unfortunately, it is not compensated for by the collection of a strong sub-bandgap current. The CB-VB recombination in the IB region-that is, $J_{l h i}+J_{s c z}+J_{r a d C V}$-is much smaller (with the exception indicated), and the emitter current is negligible.

It is interesting to observe that at low temperature a separation between the $\mathrm{CB}$ and the IB QFLs (the $V_{I C}$ voltage) actually appears. It has been indicated that when this starts to happen, the current slope (in a semi-logarithmic plot) falls below $e / k T$. This is clearly visible in Fig. 6 for all the experimental IB curves at low temperature (follow the dashed light gray line as a reference for the $e / k T$ slope). This slope is a very clear signature of QFL splitting.

TABLE IV. Current components of the IB cell for total current of $0.08 \mathrm{~A} \mathrm{~cm}^{2}$ and several temperatures.

\begin{tabular}{lcccc}
\hline \hline Current $\left(\mathrm{A} \mathrm{cm}^{-2}\right)$ & $\mathrm{T}=20 \mathrm{~K}$ & $\mathrm{~T}=77 \mathrm{~K}$ & $\mathrm{~T}=150 \mathrm{~K}$ & $\mathrm{~T}=298 \mathrm{~K}$ \\
\hline$V(\mathrm{~V})$ & 1.49039 & 1.34698 & 1.10885 & 0.74295 \\
$J_{\mathrm{em}}$ & $3.92 \times 10^{-5}$ & $3.72 \times 10^{-7}$ & $1.64 \times 10^{-8}$ & $2.08 \times 10^{-6}$ \\
$J_{\mathrm{IB}}$ & 0.07996 & 0.08000 & 0.08000 & 0.08000 \\
Sum (total $J)$ & 0.08000 & 0.08000 & 0.08000 & 0.08000 \\
$J_{\text {lhi }}$ & $1.49 \times 10^{-5}$ & 0.00205 & $5.55 \times 10^{-4}$ & $6.93 \times 10^{-4}$ \\
$J_{\text {scz }}$ & $5.11 \times 10^{-17}$ & $3.95 \times 10^{-7}$ & $1.26 \times 10^{-5}$ & 0.02753 \\
$J_{\text {radCV }}$ & 0.02318 & $4.28 \times 10^{-7}$ & $3.13 \times 10^{-9}$ & $5.31 \times 10^{-8}$ \\
$J_{\mathrm{QD}}$ & 0.05676 & 0.07795 & 0.07943 & 0.07655 \\
Sum $\left(J_{\mathrm{IB}}\right)$ & 0.07996 & 0.08000 & 0.08000 & 0.08000 \\
$V_{\mathrm{IC}}(\mathrm{V})$ & 0.24816 & 0.17502 & 0.05708 & $1.02 \times 10^{-5}$ \\
$J_{01 \mathrm{IV}}$ & 0.00176 & 0.07670 & 0.07104 & 0.01878 \\
$J_{\text {radIV }}$ & 0.05501 & 0.00112 & $1.76 \times 10^{-4}$ & $1.23 \times 10^{-5}$ \\
$J_{\text {O2IV }}$ & $1.06 \times 10^{-14}$ & $1.29 \times 10^{-4}$ & 0.00821 & 0.05776 \\
Sum $\left(J_{\mathrm{QD}}\right)$ & 0.05676 & 0.07795 & 0.07943 & 0.07655 \\
\hline \hline
\end{tabular}

\section{CONCLUSIONS}

We present a model based on standard device theory for the voltage and temperature dependence of InAs/GaAs QD IB solar cells that is also applied to ordinary GaAs test cells. The results describe the experiments with reasonable accuracy. Leakage at low voltages cannot be interpreted by this model, and we suspect that it is sample dependent. Negligible currents in the tables are in most cases estimated. Actually, in this paper we are more interested in describing the processes of recombination than the specific values of the parameters.

In order to interpret the IB solar cells, we have been forced to revisit the SRH theory of recombination through traps. We have assumed that the traps causing recombination via the successive capture of VB holes and $\mathrm{CB}$ electrons are now equally active in capturing VB holes and electrons from the IB. The very important difference is that in the IB solar cell, the main voltage and temperature dependence (associated with both radiative and non-radiative recombination) is $\exp \left(\left(e V-E_{H}\right) / k T\right)$, with $E_{H}$ being the IB-VB bandgap, whereas in ordinary cells it is $\exp \left(\left(e V-E_{g}\right) / k T\right)$. In other words, for its operation the cell bandgap has been reduced to $E_{H}$. The CB-VB recombination remains but is seldom dominant.

It is interesting to consider that, given the small concentration of minority holes, the IB-VB recombination is ruled by the hole lifetime, the same parameter that rules the $\mathrm{CB}$ VB recombination (the difference lies only in the carrier minority density, which is increased by the reduced operational bandgap). The electron lifetime differs for the CB-VB and IB-VB recombinations, but it does not affect them. It only has to be small enough to avoid hindering the recombination process, and this is actually the case according to our fitting.

The preceding statements are true for high voltages. At lower bias, the voltage and temperature dependence are of the type $\exp \left(\left(e V-E_{H}\right) / 2 k T\right)$ or $\exp \left(\left(e V-E_{g}\right) / 2 k T\right)$, and the effective lifetime is twice the geometrical mean of the hole and electron lifetimes. This allows the IB electron lifetime to be obtained from experiments.

All of the above is true provided $V_{I C}$ is negligible. At very high voltage or low temperature, however, it becomes relevant, and the importance of the recombination through the IB decreases in favor of the $\mathrm{CB}-\mathrm{VB}$ recombination, which is smaller.

The exponential pre-factor of the $\mathrm{SRH}$ recombination is also temperature dependent and decreases faster than the pre-factor for radiative recombination with decreasing temperature. Therefore, at very low temperature the latter dominates. Again, the exponential factors are those mentioned above (with either the $E_{H}$ or the $E_{g}$ bandgaps). Parameters concerning radiative recombination can be compared to absorption, and reasonable explanations can be found.

Our modeling allows for a clear determination of whether a cell is operating as an IB solar cell or not. As indicated in the Introduction and in many references on the topic, ${ }^{1,2}$ the IB solar cell has to fulfill two requirements: it must absorb sub-bandgap photons, and it must deliver the generated current at a voltage that exceeds the IB-VB 
bandgap. The first requirement is achieved and frequently cited in the literature, ${ }^{7,12,37,38}$ although unfortunately the sub-bandgap absorption is very weak. The second argument is easily verified in our model by checking the voltage $V_{I C}$ that represents the splitting of the IB and the CB QFLs. If it is zero, our effort has reduced only the bandgap, but if it is positive, it will reduce the voltage applied to the IB-VB recombination for a given external voltage, and eventually the $\mathrm{CB}-\mathrm{VB}$ recombination will become dominant, rendering the cell's voltage as good as that without IB, but benefiting from a sub-bandgap current. Now, if we look at the simulated cell, we observe that at room temperature $V_{I C}$ is zero almost always, but at voltages above $V_{o c}=0.902 \mathrm{~V}$ (the open circuit voltage at about 1000 suns), $V_{I C}$ becomes noticeable, being $V_{I C}=0.013 \mathrm{~V}$ at $V_{o c}=0.982 \mathrm{~V}$, corresponding to a concentration of about 10000 suns

As soon the temperature is reduced, $V_{I C}$ starts to present positive values at almost any voltage, showing that the cell behaves as an authentic IB solar cell. Even more, at very low temperature, the measured $V_{o c}$ is higher than the one in the test cell, as has been published elsewhere. ${ }^{11,18}$ However, it has to be said that this improved behavior is not a general property but probably due to the differences in the design of the test and IB cells (the former has a heavily doped base) or to some technological contingency.

Thus, to summarize, we have developed a reasonable model for studying the IB solar cell at different regimes of voltage and temperature. We have identified the main cause of voltage reduction of this cell at room temperature as the operational reduction of the semiconductor bandgap to the IB-VB bandgap, and we have revealed that our IB cells exhibit voltage preservation as desired when the temperature is lowered or when the voltage is high enough.

\section{ACKNOWLEDGMENTS}

This work has been supported by the IBPOWER project funded by the European Commission (Grant No. E080910B010), by the Spanish National Research Programs DenQuIBand (No. PLE2009-0045) and NANOGEFES (No. ENE2009-14481-C02-02), and by the NUMANCIA project (No. M090910B01L), funded by the Comunidad de Madrid. I. Ramiro acknowledges the Universidad Politécnica de Madrid for funding his doctoral research.

\footnotetext{
${ }^{1}$ A. Luque and A. Martí, Phys. Rev. Lett. 78, 5014 (1997).

${ }^{2}$ A. Luque and A. Martí, Prog. Photovoltaics 9, 73 (2001).

${ }^{3}$ A. Luque and A. Martí, Phys. Rev. B 55, 6994 (1997).

${ }^{4}$ A. Luque, A. Martí, and L. Cuadra, IEEE Trans. Electron Devices 48, $2118(2001)$.

${ }^{5}$ A. Martí, L. Cuadra, and A. Luque, in Proceedings of the 28th IEEE Photovoltaics Specialists Conference (IEEE, New York, 2000), pp. 940-943, 15-22 September 2000.

${ }^{6}$ W. Shockley and H. J. Queisser, J. Appl. Phys. 32, 510 (1961).
}

${ }^{7}$ A. Luque, A. Martí, C. Stanley, N. López, L. Cuadra, D. Zhou, and A. Mc-Kee, J. Appl. Phys. 96, 903 (2004).

${ }^{8}$ S. M. Hubbard, C. D. Cress, C. G. Bailey, R. P. Raffaelle, S. G. Bailey, and D. M. Wilt, Appl. Phys. Lett. 92, 123512 (2008).

${ }^{9}$ V. Popescu, G. Bester, M. C. Hanna, A. G. Norman, and A. Zunger, Phys. Rev. B 78, 205321 (2008).

${ }^{10}$ S. A. Blokhin, A. V. Sakharov, A. M. Nadtochy, A. S. Pauysov, M. V. Maximov, N. N. Ledentsov, A. R. Kovsh, S. S. Mikhrin, V. M. Lantratov, S. A. Mintairov, N. A. Kaluzhniy, and M. Z. Shvarts, Semiconductors 43, 514 (2009).

${ }^{11}$ E. Antolín, A. Martí, P. G. Linares, I. Ramiro, E. Hernández, C. D. Farmer, C. R. Stanley, and A. Luque, in Proceedings of the 35th IEEE Photovoltaic Specialists Conference (IEEE, New York, 2010), pp. 000065-000070, 20-25 June 2010.

${ }^{12}$ E. Antolín, A. Marti, C. D. Farmer, P. G. Linares, E. Hernández, A. M. Sánchez, T. Ben, S. I. Molina, C. R. Stanley, and A. Luque, J. Appl. Phys. 108, 064513 (2010).

${ }^{13}$ I. Ramiro, E. Antolin, P. G. Linares, E. Hernández, A. Martí, A. Luque, C. D. Farmer, and C. R. Stanley, Energy Procedia 10, 117 (2011).

${ }^{14}$ A. S. Lin and J. D. Phillips, IEEE Trans. Electron Devices 56, 3168 (2009).

${ }^{15}$ A. Martí, L. Cuadra, and A. Luque, IEEE Trans. Electron Devices 49, 1632 (2002).

${ }^{16}$ R. Strandberg and T. W. Reenaas, Prog. Photovoltaics 19, 21 (2011).

${ }^{17}$ K. Yoshida, Y. Okada, and N. Sano, in Proceedings of the 35th IEEE Photovoltaic Specialists Conference (IEEE, New York, 2010), pp. 000071-000075, 20-25 June 2010.

${ }^{18}$ P. G. Linares, A. Martí, E. Antolín, C. D. Farmer, I. Ramiro, C. R. Stanley, and A. Luque, Sol. Energy Mater. Sol. Cells 98, 240 (2012).

${ }^{19}$ E. Antolín and I. Ramiro, "Quantum dot solar cell exhibits intermediate band behavior at low temperatures" (unpublished).

${ }^{20}$ A. Martí, L. Cuadra, and A. Luque, IEEE Trans. Electron Devices 48, 2394 (2001)

${ }^{21}$ A. Luque, Solar Cells and Optics for Photovoltaic Concentration: Nonimagining Optics and Static Concentration (Adam Hilguer, Bristol, England, 1989), p. 308.

${ }^{22} \mathrm{~K}$. W. Boer, Survey of Semiconductor Physics (Van Nostrand Reinhold, New York, 1990).

${ }^{23}$ C. H. Henry and D. V. Lang, Phys. Rev. B 15, 989 (1977).

${ }^{24}$ A. Luque, A. Martí, E. Antolín, and C. Tablero, Physica B 382, 320 (2006).

${ }^{25}$ A. S. Grove, Physics and Technology of Semiconductor Devices (Wiley, New York, 1967).

${ }^{26}$ S. M. Sze, Physics of Semiconductor Devices (John Wiley \& Sons, New York, 1981)

${ }^{27}$ R. N. Hall, Phys. Rev. 87, 387 (1952).

${ }^{28}$ W. Shockley and W. T. Read, Jr., Phys. Rev. 87, 835 (1952).

${ }^{29}$ J. S. Wang, J. F. Chen, J. L. Huang, P. Y. Wang, and X. J. Guo, Appl. Phys. Lett. 77, 3027 (2000).

${ }^{30}$ W. van Roosbroeck and W. Shockley, Phys. Rev. 94, 1558 (1954).

${ }^{31}$ G. L. Araujo and A. Marti, Sol Energy Mater. Sol. Cells 33, 213 (1994).

${ }^{32}$ P. G. Linares, A. Marti, E. Antolin, and A. Luque, J. Appl. Phys. 109, 014313 (2011).

${ }^{33}$ A. Marti, E. Antolin, E. Canovas, N. Lopez, P. G. Linares, A. Luque, C. R. Stanley, and C. D. Farmer, Thin Solid Films 516, 6716 (2008).

${ }^{34}$ E. Cánovas, A. Martí, N. López, E. Antolín, P. G. Linares, C. D. Farmer, C. R. Stanley, and A. Luque, Thin Solid Films 516, 6943 (2008).

${ }^{35}$ A. Luque, A. Marti, E. Antolin, and P. Garcia-Linares, Sol. Energy Mater. Sol. Cells 94, 2032 (2010).

${ }^{36}$ A. Luque, P. G. Linares, E. Antolín, E. Cánovas, C. D. Farmer, C. R. Stanley, and A. Martí, Appl. Phys. Lett. 96, 013501 (2010).

${ }^{37}$ A. Luque and A. Marti, Adv. Mater. 22, 160 (2009).

${ }^{38}$ A. Martí, E. Antolin, C. R. Stanley, C. D. Farmer, N. Lopez, P. Diaz, E. Canovas, P. G. Linares, and A. Luque, Phys. Rev. Lett. 97, 247701 (2006). 